\title{
Wildlebende Arten im Palmengarten und im Botanischen Garten Frankfurt
}

\author{
Marco Schmidt, Hilke Steinecke, Andreas König,
}

Elke Brude, Klaus Dühr, Bernd Jakobus, Peter Roth, Gisela Hawickhorst,

Hermine Lotz-Winter \& Meike Piepenbring

\begin{abstract}
Botanical gardens are urban habitats rich in structure and species. In order to obtain a comprehensive overview of spontaneous species diversity, records of wild species in the Palmengarten and Botanic Garden Frankfurt were compiled from various sources and evaluated. To date, 616 wild species are known from the Palmengarten and 1897 from the Botanic Garden, including numerous Red List species. The results underline the importance of the gardens for urban biodiversity.
\end{abstract}

\section{Zusammenfassung}

Botanische Gärten sind struktur- und artenreiche städtische Lebensräume. Um eine umfassende Übersicht spontaner Artenvielfalt zu erhalten, wurden Nachweise wildlebender Arten im Palmengarten und im Botanischen Garten Frankfurt aus verschiedenen Quellen zusammengeführt und ausgewertet. Im Palmengarten sind bis jetzt 616, im Botanischen Garten 1897 wildlebende Arten bekannt, darunter auch zahlreiche Rote-Liste-Arten. Die Ergebnisse unterstreichen die Bedeutung der Gärten für die urbane Artenvielfalt.

Städtische Lebensräume sind in den vergangenen Jahrzehnten in den Fokus wissenschaftlichen und gesellschaftlichen Interesses gerückt. Dies hängt mit einer Vielzahl verschiedener Faktoren zusammen, von der noch immer fortschreitenden Urbanisierung über die Bedeutung als Refugium innerhalb des intensiv genutzten ländlichen Raums bis hin zum unmittelbaren Einfluss

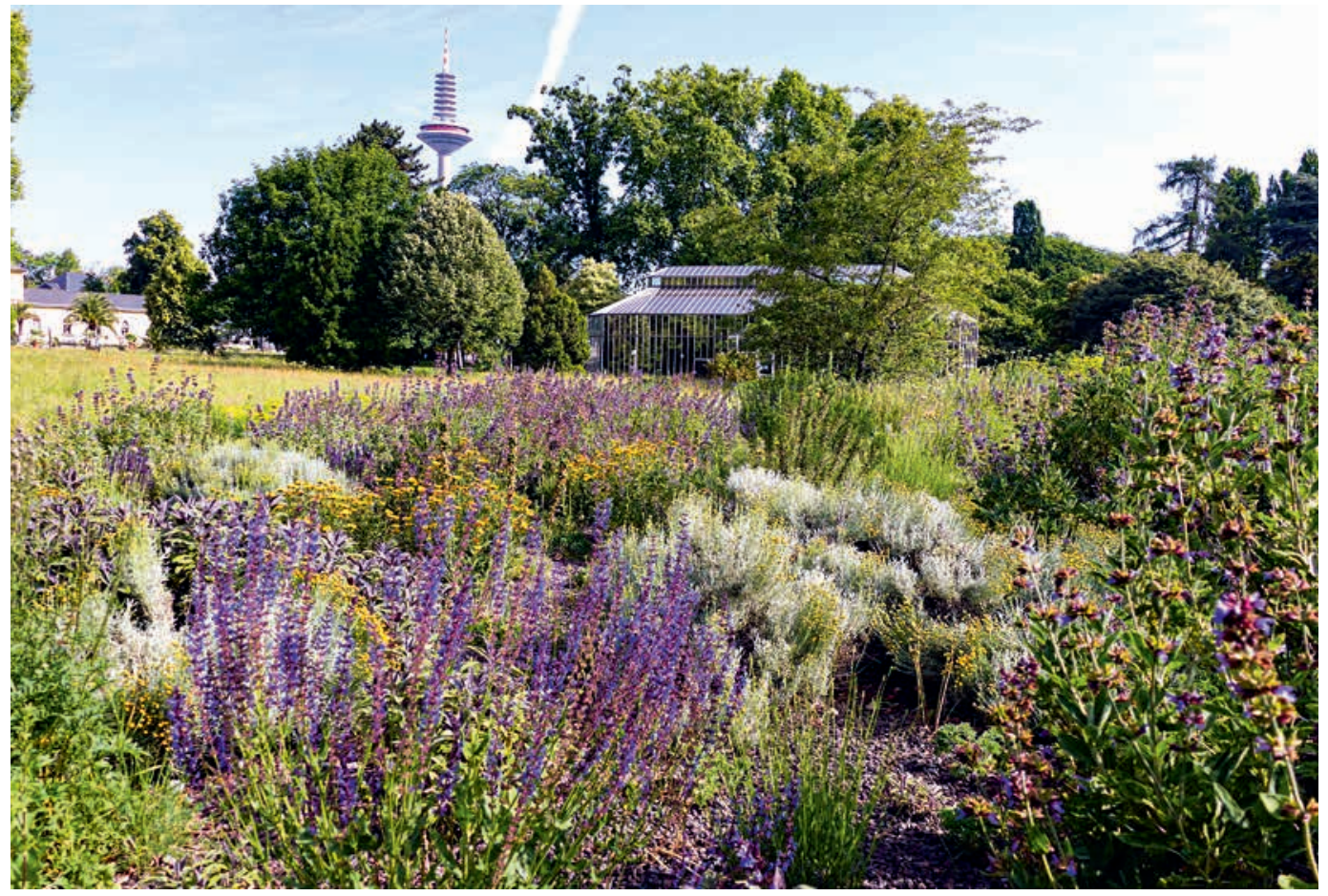

Abb. 1: Die Steppenwiese im Palmengarten wird mit ihren zahlreichen Blüten von vielen Hautflüglern und Schmetterlingen besucht. (Foto: H. Steinecke) 


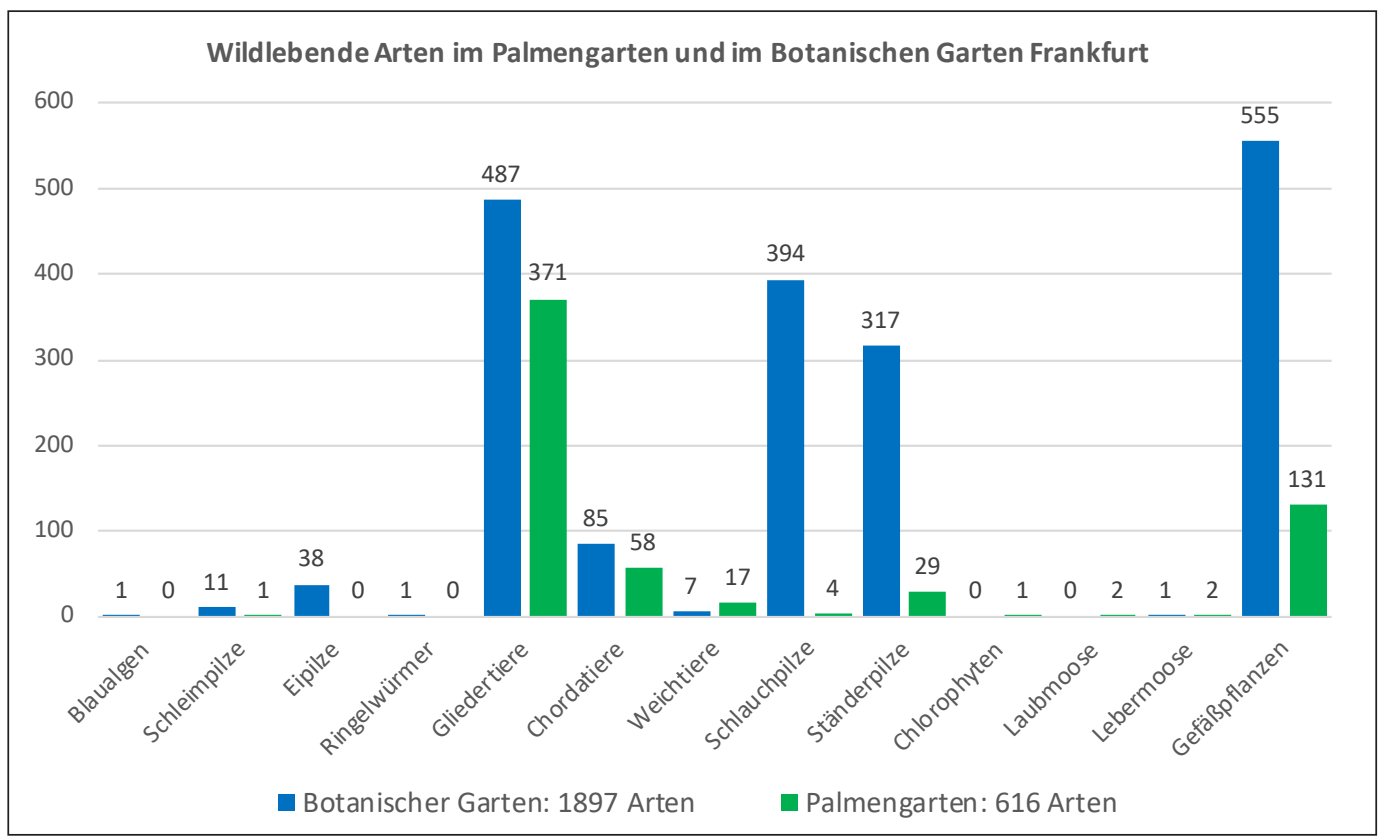

Abb 2 : Beobachtete Arten nach Stamm/Abteilung: Gliedertiere (der größte Teil davon Insekten), Chordatiere (hier ausschließlich Wirbeltiere, v.a. Vögel) und Gefäßpflanzen wurden in beiden Gärten in großer Zahl erfasst, für Pilze und pilzähnliche Organismen liegen vor allem Daten aus dem Botanischen Garten, zu anderen Organismen bislang nur Einzelbeobachtungen vor. (Grafik: M. SснміDт)

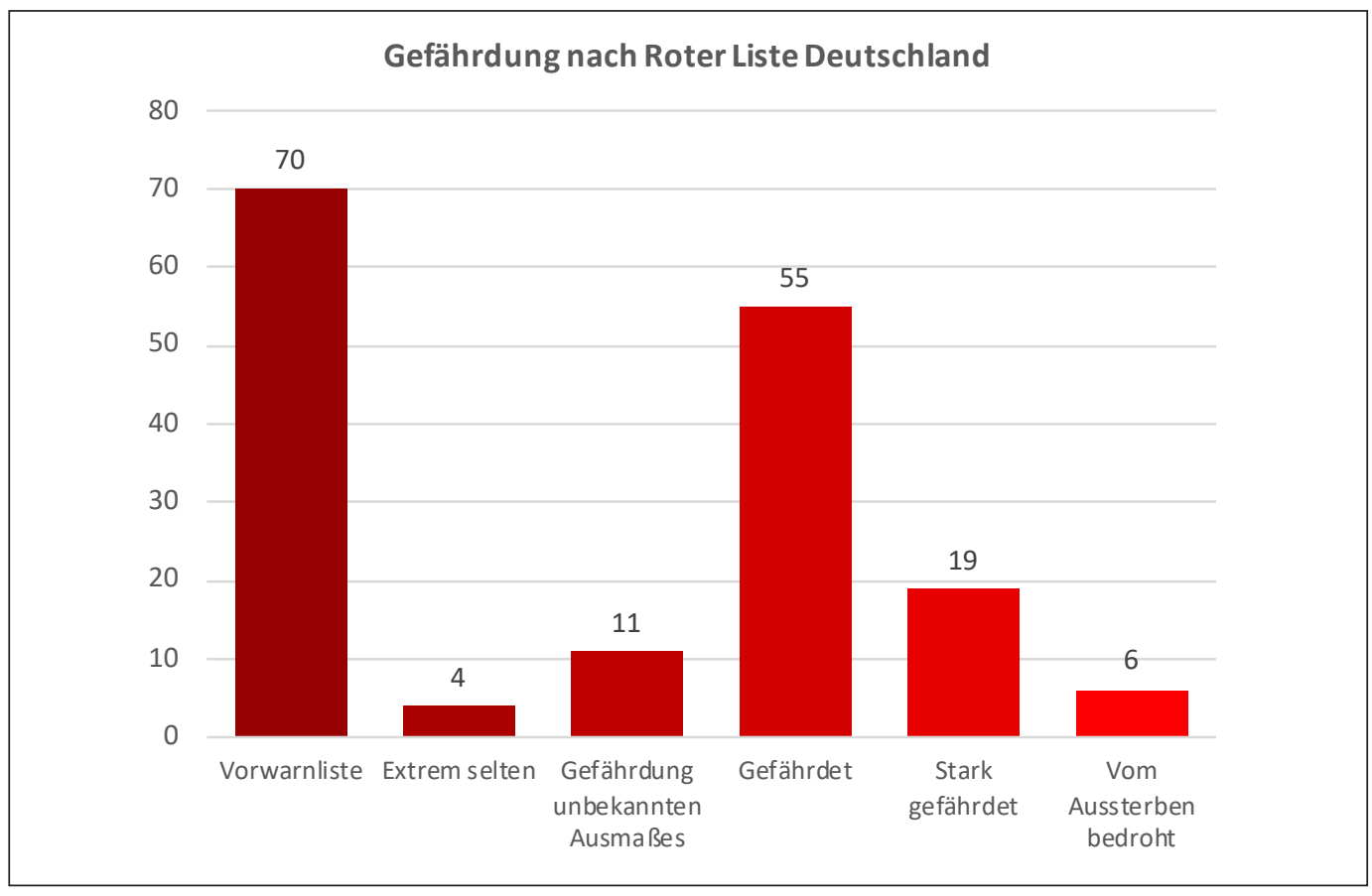

Abb. 3: Spontan vorkommende Rote-Liste-Arten im Botanischen Garten Frankfurt nach Gefährdungskategorien. (Grafik: M. SснміDт) 


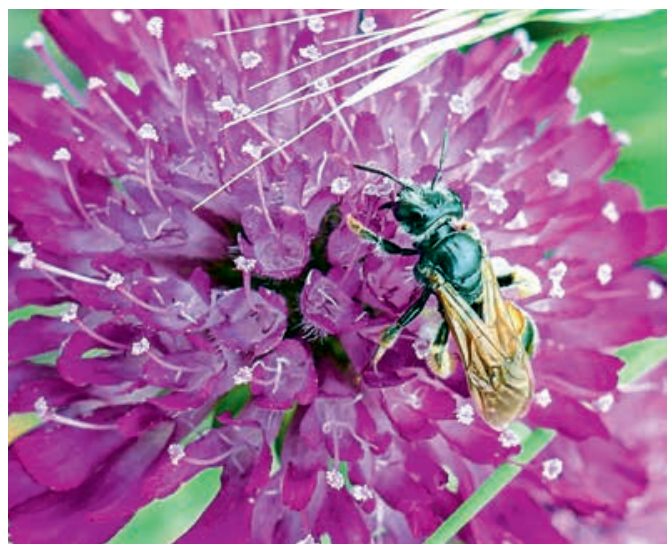

Abb. 4 : Knautien-Sandbiene (Andrena hattorfiana), eine gefährdete Art nach Roter Liste Deutschland.

(Foto: H. Steinecke)

von Stadtgrün und begleitender Biodiversität auf unser Wohlbefinden. Für die Stadt Frankfurt ist kürzlich eine umfassende Zusammenstellung der "Stadtnatur" erschienen (STARKe-OtTich \& Zizka 2019), die viele artenreiche, naturnahe und renaturierte Lebensräume sowie verschiedene Organismengruppen umfasst. Das kurz vor der Fertigstellung befindliche Arten- und Biotopschutzkonzept der Stadt Frankfurt soll die „Stadtnatur" auch im politischen Rahmen verankern. Vom Verband der Botanischen Gärten wird eine Übersicht der wildlebenden Arten in botanischen Gärten in Deutschland, Österreich und der Schweiz vorbereitet. In diesem $\mathrm{Zu}$ sammenhang haben wir für die beiden innerstädtischen Gärten in Frankfurt alle bekannten Nachweise spontan vorkommender Organismen zusammengestellt.

Die uns vorliegenden Biodiversitätsdaten stammen aus wissenschaftlichen Veröffentlichungen (De Armas \& Rehfeldt 2015, Decker et al. 2014, Kruse et al. 2020, Lotz-Winter et al. 2011), Diplom- und Examensarbeiten (HeussLER 2009, MaIER 1992), einem Gartenführer zum Botanischen Garten (Jenny et al. 2014), unveröffentlichten Artenlisten des Senckenberg-„Flechtenkränzchens“ (Beobachtungen 2009-2021 von Marion Eichler, Rainer Cezanne, Christian Printzen, Marie-Luise Hohmann, Ute Wernicke, Fernando Fern-

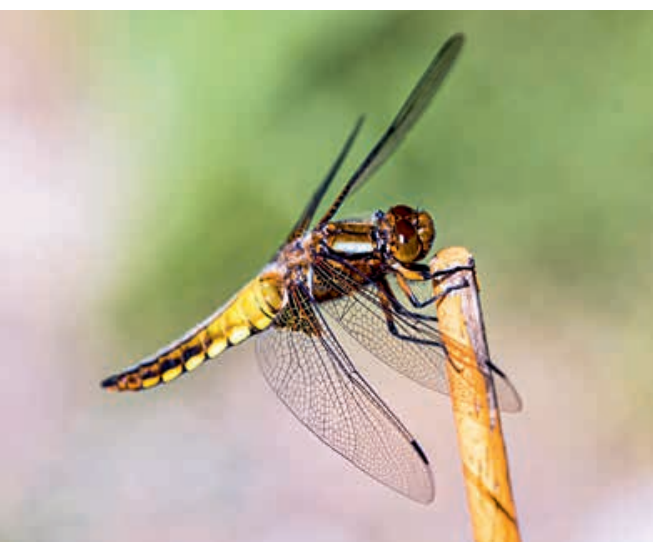

Abb. 5: Junges Weibchen des Plattbauchs (Libellula depressa). (Foto: G. НаwicкHоRsт)

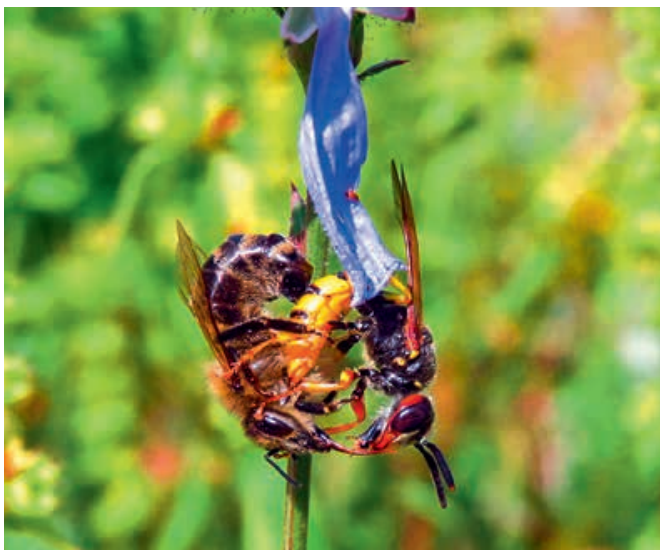

Abb. 6: Ein Bienenwolf (Philanthus triangulum) mit erbeuteter Biene. (Foto: P. Roth)

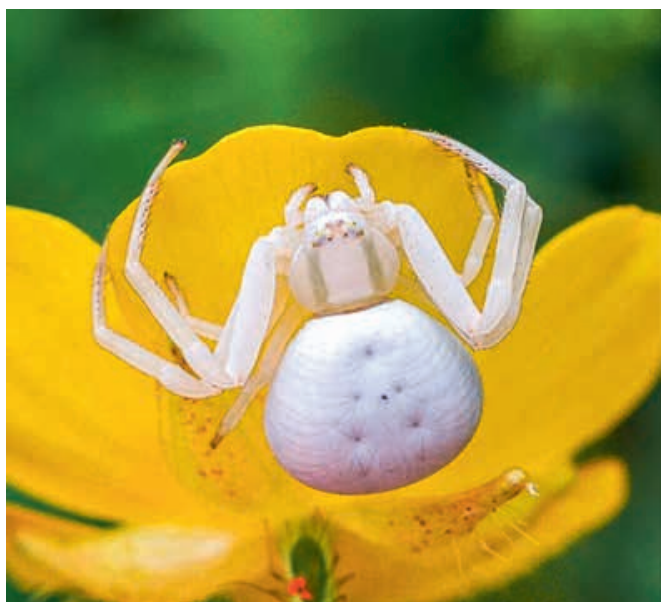

Abb. 7: Veränderliche Krabbenspinne (Misumena vatia). (Foto: K. DüHR). 


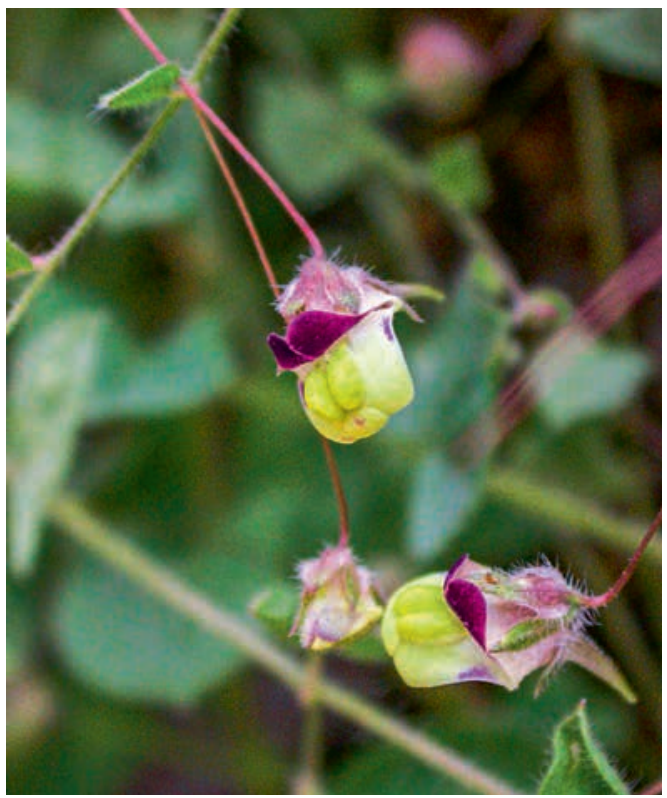

Abb. 8: Echtes Tännelkraut (Kickxia elatine, Rote Liste Deutschland 3). (Foto: E. BRUdE)

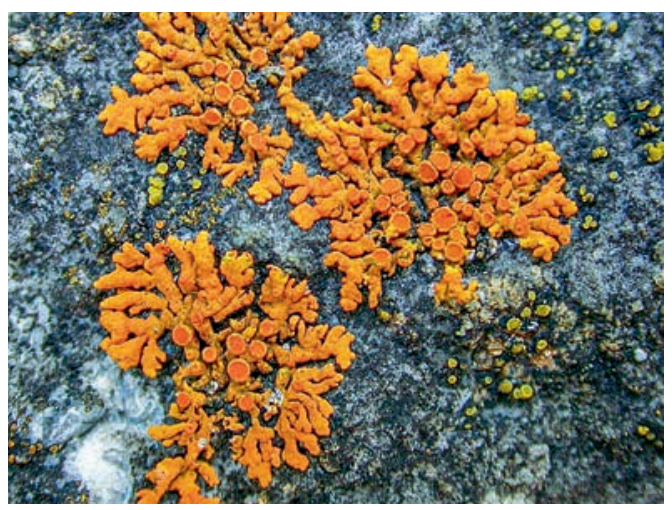

Abb. 9: Zierliche Gelbflechte (Rusavskia elegans). (Foto: E. BRUde)

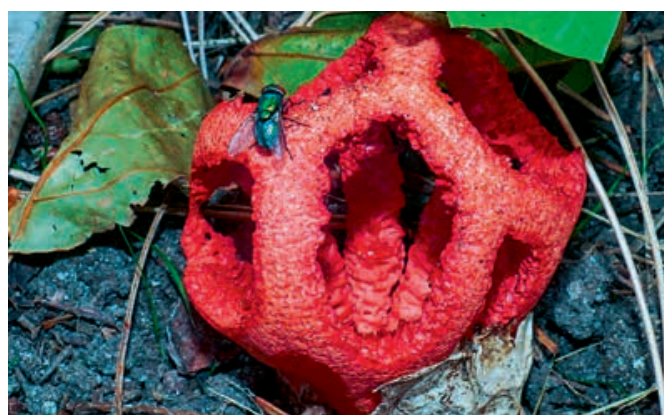

Abb. 10: Roter Gitterpilz (Clathrus ruber).

(Foto: H. Steinecke) andez Mendoza und Elke Brude) und von einem Bürgerwissenschaftsprojekt auf der iNaturalist-Plattform (SснміDт et al. 2019). Daten aus diesen unterschiedlichen Quellen wurden in einer relationalen Datenbank zusammengeführt und zur Harmonisierung der Nomenklatur und Synonymie mit dem Species Matching Tool (https://www.gbif.org/tools/species-lookup) der in der Global Biodiversity Information Facility (GBIF) verwendeten Taxonomie (GBIF Secretariat 2019) angepasst. So wird verhindert, dass eine Art unter verschiedenen Namen mehrfach gezählt wird. Unterarten und Varietäten wurden auf Artebene zusammengefasst, um eine Vergleichbarkeit zu gewährleisten.

Die Auswertungen für beide Gärten zeigen die Bedeutung der Gärten als Lebensraum für zahlreiche wildlebende Arten, machen aber ebenso deutlich, wie groß die Lücken in der Erfassung noch sind. Im Botanischen Garten wurden mehr als drei Mal so viele Arten nachgewiesen, wie im Palmengarten. Das hängt zum einen mit der langjährigen Universitätsgeschichte des Botanischen Gartens zusammen, die Erfassungen durch die Wissenschaftler der benachbarten Institute begünstigte, zum anderen auch durch die hier naturnah dargestellten Lebensräume mit einer Vielzahl heimischer Pflanzenarten, die für heimische Tiere und Pilze beste ökologische Voraussetzungen bietet.

Während die Pilze im Botanischen Garten Frankfurt durch Lotz-Winter et al. (2011) und KRUSE et al. (2020) so gut erfasst sind, wie in kaum einem anderen Botanischen Garten Mitteleuropas, wurden andere Organismengruppen bislang ausschließlich durch das Engagement der Bürgerwissenschaftler in unseren Gärten erfasst. Das ist der Fall z.B. für Wildbienen, die in München, Dresden und Berlin (Bembé et al 2001, MüNZE et al. 2006, SAURE 2012) erfasst wurden, kaum jedoch für die Gärten in Frankfurt. Durch systematische wissenschaftliche Untersuchungen würden hier sicher noch zahlreiche weitere Arten nachgewiesen werden. Wie im Botanischen Garten stammen auch im Palmengarten die meisten Artnachweise aus den Freilandbereichen, nur 


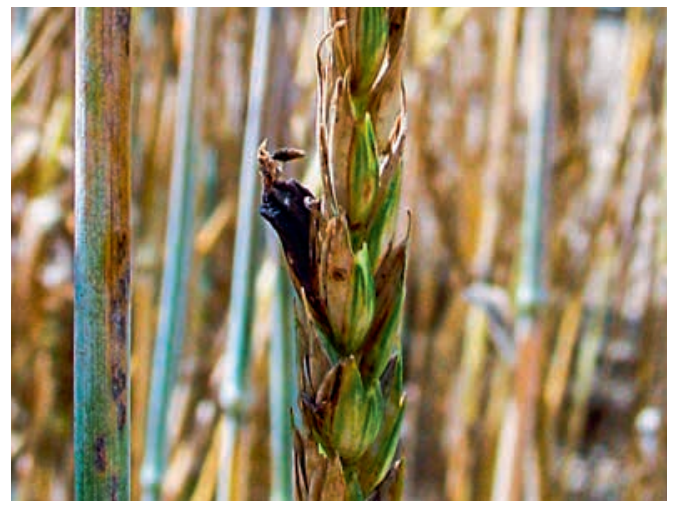

Abb. 11: Sklerotien des Mutterkornpilzes (Claviceps purpurea) auf $\times$ Secalotricum. (Foto: M. Piepenbring)

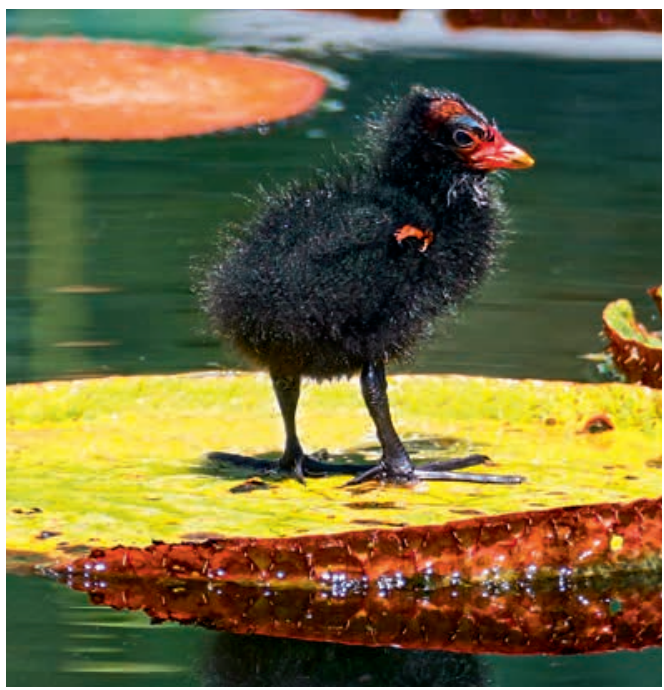

Abb. 13: Teichhuhn (Gallinula chloropus) auf einem Blatt der Riesen-Seerose (Victoria cruziana). (Foto: H. Steinecke)

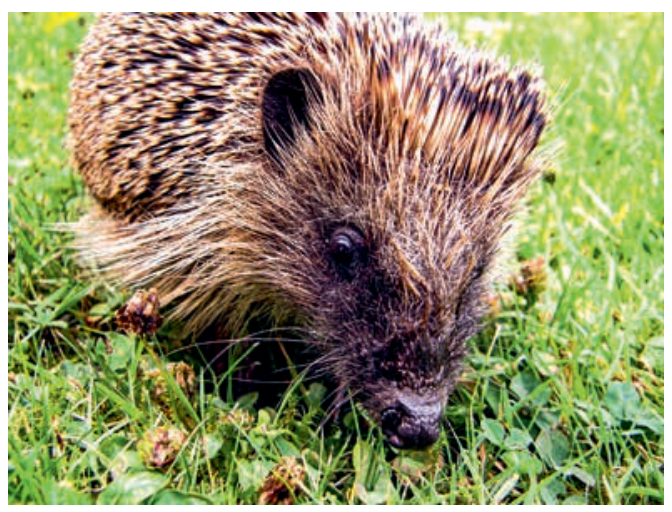

Abb. 15: Westeuropäischer Igel (Erinaceus europaeus).

(Foto: H. Steinecke)

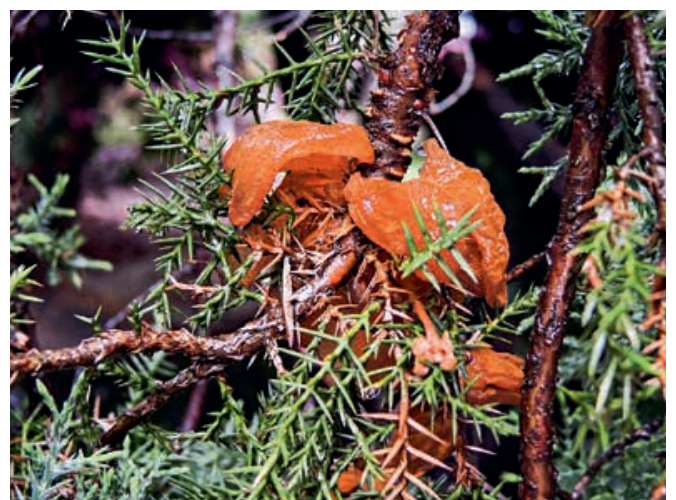

Abb. 12: Birnengitterrost (Gymnosporangium sabinae) auf Juniperus sp. (Foto: M. Piepenbring)

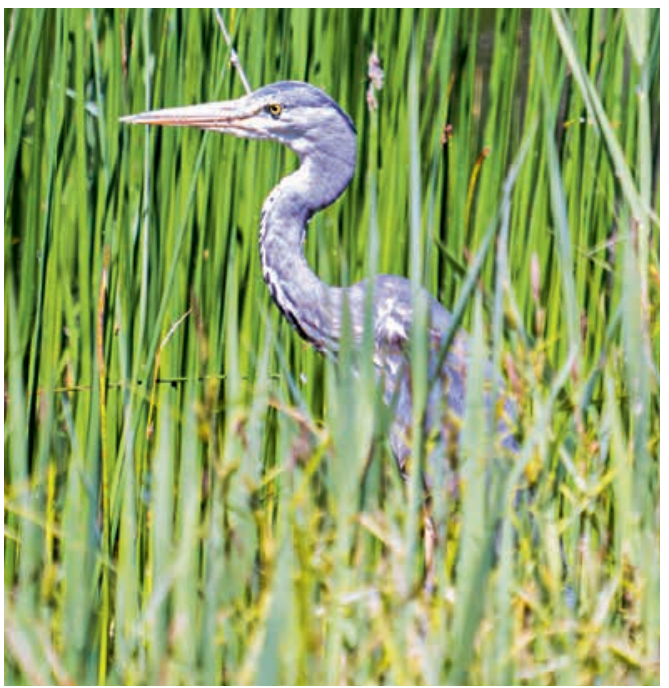

Abb. 14: Graureiher (Ardea cinerea). (Foto: G. HawickHORST)

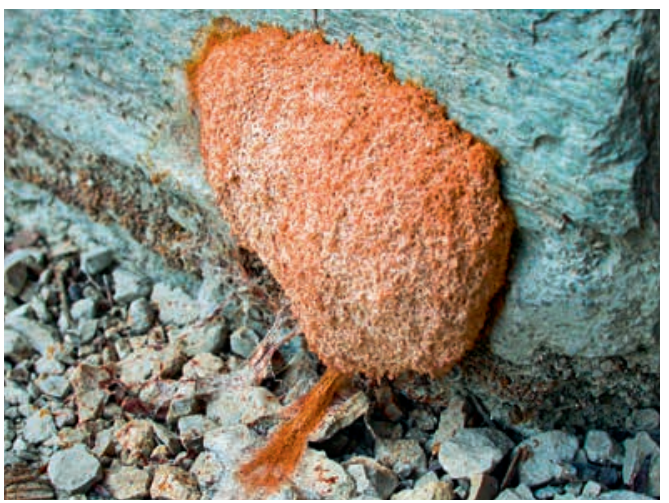

Abb. 16: Die rotbraune Varietät der Gelben Lohblüte (Fuligo septica var. rufa). (Foto: E. BRUDE) 


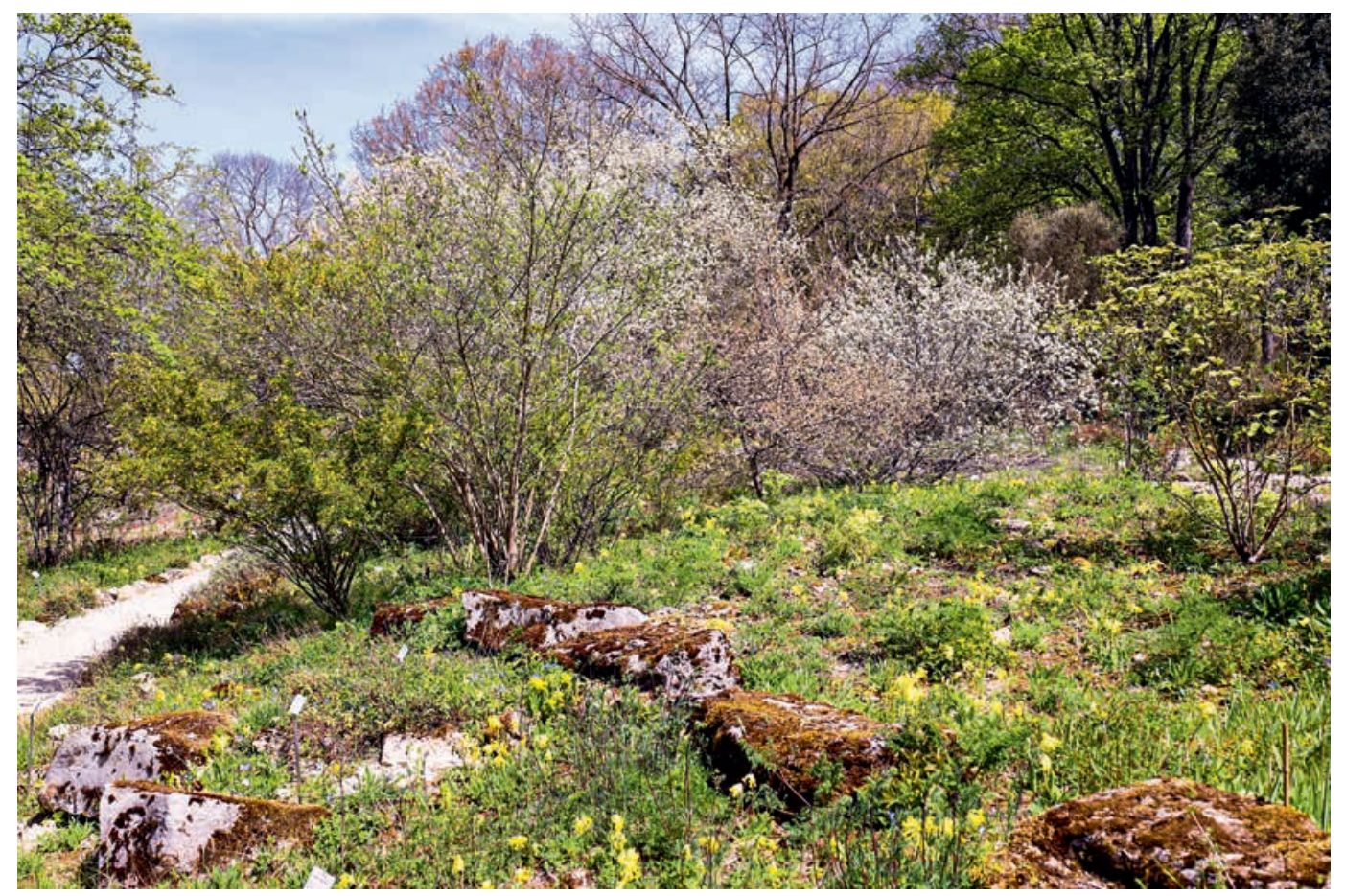

Abb. 17: Der Kalktrockenhang im Botanischen Garten ist strukturreich und artenreich bepflanzt, so dass er vielen wildlebenden Arten ein geeignetes Habitat bietet. (Foto: H. Steinecke)

wenige aus den Gewächshäusern. Besonders erwähnenswert ist dabei ein Geißelskorpion, der im Palmengarten in den Gewächshäusern entdeckt wurde und den Garten im Namen trägt: Bucinozomus hortuspalmarum (ARMAS \& REHFELDT 2015).

Im Botanischen Garten kommen zahlreiche Arten vor, deren Gefährdung in den Roten Listen Deutschlands (www.rote-liste-zentrum.de) dokumentiert ist. Der größte Teil dieser Arten sind Samenpflanzen, die bewusst eingebracht wurden, sich aber spontan im Garten vermehren und ausbreiten. Auch unter den Flechten und Insekten gibt es mehrere gefährdete Arten, die hier einen Lebensraum gefunden haben, in anderen Organismengruppen nur einzelne Vertreter.

Eine komplette tabellarische Zusammenstellung aller uns bekannten wildlebenden Arten unserer Gärten inklusive Rote-Liste-Kategorien und systematischer Zugehörigkeit findet sich als Anhang der digitalen Version dieses Artikels.

\section{Literatur}

Bembé, B., Gerlach, G., Schuberth, J. \& Schönitzer, K. 2001. Die Wildbienen im Botanischen Garten München. Nachrichtenblatt der bayerischen Entomologen 50: 30-41.

De Armas, L. F., \& Rehfeldt, S. 2015: Stenochrus portoricensis, Zomus bagnallii and a new genus of schizomids (Schizomida: Hubbardiidae) from a greenhouse in Frankfurt am Main, Germany. - Arachnologische Mitteilungen 49: 55-61.

Decker P, Reip, H., Voigtränder, K. 2014: Millipedes and centipedes in German greenhouses (Myriapoda: Diplopoda, Chilopoda). - Biodiversity Data Journal 2: e1066.

GBIF Secretariat 2019: GBIF Backbone Taxonomy. - Checklist dataset. https://doi.org/10.15468/39omei

Heussler, E. 2009: Untersuchungen zu den Biotoptypen und zur epigäischen Fauna des Palmengartens der Stadt Frankfurt am Main. 110 S. Diplomarbeit Goethe-Universität. Frankfurt am Main.

Jenny, M., Wessel, M. \& Winter, C. Hrsg. 2014: Der Botanische Garten Frankfurt am Main. Ein illustrierter Führer. - Books on Demand, Norderstedt.

Kruse, J., Lotz-Winter. H., Piepenbring, M. \& SanDAU, H. 2020: Fungi of the Botanical Garden Frankfurt am Main - Contribution to the diversity of fungi and fungus-like organisms. - Zeitschrift für Mykologie 86: 37-76. 


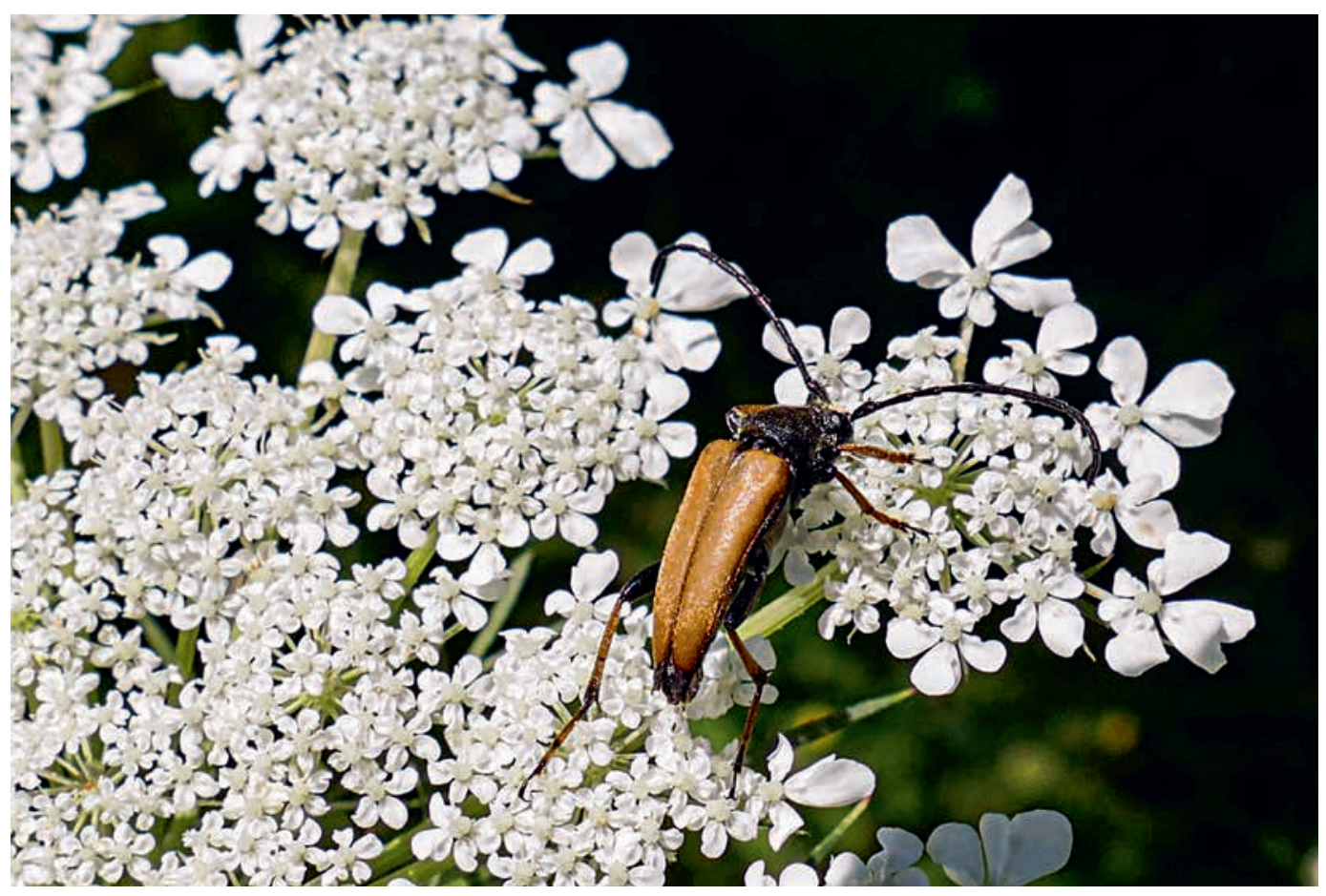

Abb. 18: Rothalsbock (Stictoleptura rubra). (Foto: H. STEINECKE)

Lotz-Winter H., Hofmann, T., Kirschner, R., Kursawe, M., Trampe, T. \& Piepenbring, M. 2011: Fungi in the Botanical Garden of the University of Frankfurt. - Zeitschrift für Mykologie 77: 89-122.

Maier, K. 1992: Faunistisch-ökologische Untersuchungen an epigäischen Arthropoden (Coleoptera: Carabidae; Araneae) in einem Stadtpark. Diplomarbeit TH Darmstadt. - Darmstadt.

Münze, R., Langner, D. \& Nuss, M. 2006: Die Bienenfauna des Botanischen Gartens Dresden (Hymenoptera: Apidae). - Sächsische Entomologische Zeitschrift 1: 45-69.

Saure, C. 2012. Die Wildbienen des Botanischen Gartens Berlin-Dahlem (Hymenoptera Apiformes). - Märkische Entomologische Nachrichten 14: 29-67.

Schmidt, M., König, A., Steinecke, H. \& Zizka, G. 2019: Tiere und Pilze in Frankfurts Botanischen Gärten - ein Bürgerwissenschaftsprojekt: https:/www.inaturalist.org/projects/tiere-und-pilze-in-frankfurts-botanischen-garten. Palmengarten. 83: 54-59.

Starke-Ottich, I. \& Zizka, G. 2019. Stadtnatur in Frankfurt - vielfältig, schützenswert, notwendig. Senckenberg-Buch 82. - Frankfurt am Main.

Anhang 1 in der online-Version: Artenliste Palmengarten Anhang 2 in der online-Version: Artenliste Botanischer Garten

\section{Anschriften der Autorinnen und Autoren}

Dr. Marco Schmidt, Palmengarten Frankfurt, Siesmayerstr. 61, 60323 Frankfurt,

E-Mail: marco.schmidt@stadt-frankfurt.de; https://orcid.org/0000-0001-6087-6117

Dr. Hilke Steinecke, Palmengarten Frankfurt, Siesmayerstr. 61, 60323 Frankfurt,

E-Mail: hilke.steinecke@stadt-frankfurt.de; https://orcid.org/0000-0002-4618-4867

Andreas König, Botanischer Garten Frankfurt, Siesmayerstr. 72, 60323 Frankfurt,

E-Mail: andreas.koenig@stadt-frankfurt.de

Elke BRude, Freundeskreis Botanischer Garten Frankfurt am Main e.V. , Siesmayerstr. 72, 60323 Frankfurt, E-Mail: elke.brude@t-online.de

Peter Roth, Palmengarten Frankfurt, Siesmayerstr. 61, 60323 Frankfurt, E-Mail: petrotffm@gmx.de

Mag. Pharm. Hermine Lotz-Winter, Goethe-Universität Frankfurt am Main, Biologicum, Max-von-Laue-Str. 13, 60438 Frankfurt am Main, E-Mail: hermine.lotz@email.de

Prof. Dr. Meike Piepenbring, Goethe-Universität Frankfurt am Main, Biologicum, Max-von-Laue-Str. 13, 60438 Frankfurt am Main,

E-Mail: piepenbring@bio.uni-frankfurt.de 